\title{
The Use of Metronidazole for Treating Parasitic Infections Improves the Periodontal Health
}

\author{
Khalid Mahmoud Ababneh* and Ali Hassan AlWaked \\ Ministry of Health, Jordan \\ *Corresponding author: Khalid Mahmoud Ababneh, Ministry of Health, Jordan \\ To Cite This Article: Khalid Mahmoud A, Ali Hassan A. The Use of Metronidazole for Treating Parasitic Infections Improves the Periodontal \\ Health. Am J Biomed Sci \& Res. 2019 - 1(5). AJBSR.MS.ID.000549. DOI: 10.34297/AJBSR.2019.01.000549
}

Received: March 06, 2019 | Published: March 11, 2019

\begin{abstract}
The present study reviewed the literature regarding the impacts of using metronidazole for treating parasitic infections in improving the periodontal health. The prevalence of chronic periodontitis varies between 15-30\% among general population. Two important aspects have been discussed, the involvement of using metronidazole treatment for other diseases such as cardiovascular diseases. The other aspect is the existence of patients with poor oral hygiene and accepted gingival health status. We put the link through the use of metronidazole for other diseases such as parasitic infections, which indirectly improves the periodontal and gingival health. As a conclusion, from a clinical point of view, patients with poor oral hygiene are expected to develop chronic periodontitis, but due to previous treatments for other diseases including parasitic infections that involve the use of metronidazole, the periodontum health has been improved. Accordingly, it is suggested to take this into consideration in assessment patients with poor oral hygiene.
\end{abstract}

Keywords: Metronidazole; Chronic Periodontitis; Gingiva; Parasitic infection; Antibiotics; Anaerobic Organisms

\section{Introduction}

Metronidazole, known as Flagyl, has therapeutic uses as antibiotic and antiprotozoal [1]. It can be used as a single treatment or combined with others to treat several inflammatory conditions such as pelvic inflammatory disease, endocarditis, and bacterial vaginosis [2]. Metronidazole can be used orally, as a cream, and intravenously [2].

The prime medical use of metronidazole is to treat bacterial vaginosis, pelvic inflammatory disease, pseudomembranous colitis, aspiration pneumonia, rosacea (topical), fungating wounds (topical), intra-abdominal infections, lung abscess, periodontitis, amoebiasis, oral infections, giardiasis, trichomoniasis, and infections caused by susceptible anaerobic organisms such as Bacteroides, Fusobacterium, Clostridium, Peptostreptococcus, and Prevotella species [3]. In the following sections, there will be a focus on periodontal health and the role of using metronidazole in treating other conditions in improving the health status of gingiva and periodontum.

\section{Periodontal Diseases}

Periodontal diseases are considered as chronic inflammations that are attributed to the infection of the gingiva (gingivitis) and underlying parts of the periodontium such as periodontitis $[4,5]$.
Periodontal diseases are characterized by their complexity and involving of interfering multifactorial agents. The etiology of periodontitis involves the existence of two components, little number of Gram-negative anaerobic bacteria in subgingival sites, and uncontrolled host reactions against such pathogens $[4,5]$. The prevalence of periodontitis is about $15 \%$ of population internationally [6]. Leaving periodontitis without treatment may lead to tooth loss and other diseases such as cardiovascular diseases and preterm low birth weight babies [7].

According to the study of Pizzo et al. [7], in recent decades, the relationship between dentistry and internal medicine and in particular the so-called theory of focal infections has long been controversial. The focal disease pathogenesis was thought to be related to pathology of dental pulp and periapical infections. Nowadays, it seems that there is an exchange of the role since there is an increasing interest in the role of periodontal infection in systemic diseases. Actually, periodontal pathogens and their related inflammatory products have the ability to enter the the circulation which can induce systemic diseases. According to this context, chronic periodontitis can be considered as a risk factor for several diseases such as cardiovascular diseases, diabetes mellitus, respiratory disease, and preterm delivery. 


\section{Metronidazole and Periodontitis}

Metronidazole is highly prescribed for the treatment of periodontal diseases because it can act against several types of Gram-negative anaerobic bacteria through the inhibition of bacterial nucleic acid synthesis [8].

Anaerobic infections are usually considered as having a polymicrobial nature. Other facultative bacteria, not susceptible to metronidazole including streptococci, Actinomyces spp., Aggregatibacter spp. and Haemophilus spp are involved. Accordingly, it is suggested to either use broad-spectrum antibiotics alone or to be used in combination to cover all potential pathogens $[9,10]$.

The frequency of using metronidazole in treating parasitic infection.

Soule et al. [11] conducted a study to assess the frequency of clinical cure among patients who received metronidazole every 12 $\mathrm{h}$ compared with those who received an every $8 \mathrm{~h}$ frequency. The results did not show significant differences in clinical cure for every $12 \mathrm{~h}$ versus every $8 \mathrm{~h}$ metronidazole dosing regimens.

Metronidazole has been used to treat various anaerobic infections [12]. Anaerobic bacteria constitute normal human flora in the oral and intestinal spaces, and become causative pathogens if infection exists at these sites [13-15].

We think that the use of metronidazole for infectious diseases improves the gingival health status, although patients exhibit poor oral hygiene. This may mask the impacts of chronic periodontitis and is expected to increase the severity of periodontitis over the time.

\section{Conclusion}

From a clinical point of view, patients with poor oral hygiene are expected to develop chronic periodontitis, but due to previous treatments for other diseases including parasitic infections that involve the use of metronidazole, the periodontum health has been improved. Accordingly, it is suggested to take this into consideration in assessment patients with poor oral hygiene.

\section{References}

1. Andrés Bendesky, Daniel Menéndez, Patricia Ostrosky Wegman (2002) Is metronidazole carcinogenic? Mutation Research 511(2): 133-144.

2. Metronidazole.

3. Rossi S Australian Medicines Handbook (2013) The Australian Medicines Handbook Unit Trust. Adelaide, Australia.

4. Pihlstrom B L, Michalowicz B S, Johnson N W (2005) Periodontal diseases. Lancet 366(9499): 1809-1820.

5. Jabrane Azelmat, Jade Fournier Larente, Daniel Grenier (2015) The anthraquinone Rhein exhibits synergistic antibacterial activity in association with metronidazole or natural compounds and attenuates virulence gene expression in Prohormones gingivalis. Archives of oral biology 60(2): 342-346.

6. Eke PI, Dye BA, Wei L, Thornton Evans G O, Genco R J (2012) Prevalence of periodontitis in adults in the Unites States: 2009 and 2010. J Dent Res 91(10): 914-920.

7. Pizzo G, Guiglia R, Russo L L, Campisi G (2010) Dentistry and internal medicine: from the focal infection theory to the periodontal medicine concept. Eur J Intern Med 21(6): 496-502.

8. A Rizzo, R Paolillo, L Guida, M Annunziata, N Bevilacqua, et al. (2010) Effect of metronidazole and modulation of cytokine production on human periodontal ligament cells. Int Immunopharmacology 10(7): 744-750.

9. A J Van Winkelhoff, J P Rodenburg, R J Goené, F Abbas, E G Winkel, et al. (1989) Metronidazole plus amoxicillin in the treatment of Actinobacillus antinomy ceremonials associated periodontitis. J Clin Periodontal 16(2): 128-131.

10. A Mombelli (2012) Antimicrobial advances in treating periodontal diseases Front. Oral Biol 15: 133-148.

11. Soule, A F, Green, S B, Blanchette, L M (2018) Clinical efficacy of 12-h metronidazole dosing regimens in patients with anaerobic or mixed anaerobic infections. Therapeutic advances in infectious disease 5(3): 57-62.

12. Lofmark S, Edlund C, Nord C E (2010) Metronidazole is still the drug of choice for treatment of anaerobic infections. Clinical Infectious Diseases 50(Suppl 1): S16-S23.

13. Brook I (2007) Microbiology and principles of antimicrobial therapy for head and neck infections. Infect Dis Clin North Am 21(2): 355-391.

14. Brook I, Wexler H and Goldstein E (2013) Antianaerobic antimicrobials: spectrum and susceptibility testing. Clin Microbiol Rev 26(3): 526-546.

15. Solomkin J S (2010) Guidelines for the selection of anti-infective agents for complicated intra-abdominal infections. Clin Infect Dis 501: 133-164. 